\title{
Resina fluida autoadhesiva utilizada como sellante de fosas y fisuras. Estudio de microinfiltración
}

\author{
Self-adhesive flowable composite-resin as a fissure sealant. A \\ microleakage study
}

\author{
De Nordenflycht $\mathrm{D}^{1}$, Villalobos $\mathrm{P}^{2}$, Buchett $\mathrm{O}^{3}$, Báez $\mathrm{A}^{4}$
}

\begin{abstract}
RESUMEN
Objetivo: Evaluar la capacidad de sellado de una resina fluida autoadhesiva (Fusio Liquid Dentin, Pentron Clinical) utilizada como sellante de fosas y fisuras con distintos acondicionamientos de la superficie de esmalte. Materiales y Métodos: Se seleccionaron 140 terceros molares recientemente extraídos, los que fueron distribuidos aleatoriamente en cuatro grupos $(n=35)$ y recibieron una técnica de acondicionamiento del esmalte y aplicación de un sellante. Se establecieron los siguientes grupos: Grupo 1, grabado ácido y aplicación de sellante (Clinpro, 3M ESPE); Grupo 2, grabado ácido y aplicación de resina autoadhesiva (Fusio Liquid Dentin, Pentron Clinical); Grupo 3, aplicación de resina autoadhesiva; Grupo 4, microarenado del esmalte y aplicación de resina autoadhesiva. Los dientes sellados fueron termociclados ( 500 ciclos, $\left.5-55^{\circ} \mathrm{C}\right)$, y posteriormente sumergidos en solución de nitrato de plata amoniacal por $24 \mathrm{~h}(\mathrm{pH}=14)$ y luego en revelador radiográfico (GBX, Kodak) por 8h. Posteriormente, los dientes fueron cortados para obtener 2 láminas por diente que fueron observadas bajo magnificación $(4 \mathrm{x})$ y analizadas digitalmente para evaluar la microinfiltración y la penetración en la fisura. Los resultados fueron analizados estadísticamente (ANOVA, Dunnett, $p<0.05$ ). Resultados: La microinfiltración del Grupo $1(13.18 \pm 9.25 \%)$ fue significativamente menor que la de los grupos 2,3 y 4 ( $p<0.05)$. La penetración en la fisura en el Grupo 1 (98.92 $\pm 2.77 \%)$ fue mayor que en los otros grupos, siendo esta diferencia significativa sólo con el Grupo 4. Conclusión: Fusio Liquid Dentin tiene una capacidad de sellado inferior a la del sellante convencional Clinpro, independiente del acondicionamiento del esmalte que se utilice.
\end{abstract}

Rev. Clin. Periodoncia Implantol. Rehabil. Oral Vol. 6(1); 5-8, 2013.

Palabras clave: Fusio Liquid Dentin, microinfiltración, resina fluida autoadhesiva, sellantes.

\section{ABSTRACT}

Aim: To evaluate the sealing ability of a self-adhesive flowable composite-resin (Fusio Liquid Dentin, Pentron Clinical) with different conditioning treatments of the enamel surface used as a fissure sealant. Materials and Method: 140 recently extracted human third molars were selected and randomly divided into four groups $(n=35)$. Each group received an enamel conditioning treatment and a sealant application. The following groups were established: Group 1, acid etching and sealant application (Clinpro, 3M ESPE); Group 2, acid etching and self-adhesive flowable composite-resin (Fusio Liquid Dentin, Pentron Clinical); Group 3, self-adhesive flowable composite-resin; Group 4, sandblasting and self-adhesive flowable compositeresin. Sealed teeth were thermocycled $\left(500\right.$ cycles, $\left.5-55^{\circ} \mathrm{C}\right)$ and immersed in ammoniacal silver nitrate solution for $24 \mathrm{~h}$ (ph=14). After that, the teeth were immersed in radiographic developer solution (GBX, Kodak) for $8 \mathrm{~h}$. Then, the teeth were vertically sectioned to obtain two slides each that were observed under magnification (4x) and digitally analyzed to assess microleakage and sealant penetration. Results were statistically analyzed (ANOVA, Dunnett, $p<0.05)$. Results: Microleakage of Group $1(13.18 \pm 9.25 \%)$ was significantly lower than groups 2,3 and 4 ( $p<0.05$ ). Group 1 had the highest sealant penetration mean $(98.92 \pm 2.77 \%)$. Conclusion: Fusio Liquid Dentin had a worse sealing ability than Clinpro irrespective of their enamel conditioning treatment when used as a fissure sealant. Among Fusio's groups, acid etching was the best enamel surface conditioning treatment.

Rev. Clin. Periodoncia Implantol. Rehabil. Oral Vol. 6(1); 5-8, 2013.

Key words: Fusio Liquid Dentin, microleakage, sealants, self-adhesive flowable resin.

\section{INTRODUCCIÓN}

Los sellantes basados en resina son una medida efectiva de control de caries en niños y adolescente, observándose reducción en la incidencia de nuevas lesiones oclusales cercana al $80 \%{ }^{(1)}$. Desde la introducción de la técnica del sellado de superficies oclusales descrita por Cueto y Buonocore en $1967^{(2)}$, los clínicos e investigadores han estudiado técnicas y materiales que permitan simplificar la técnica y mejorar la efectividad del procedimiento. Debido a esto, se han desarrollado sellantes basados en ionómeros de vidrio, en resina de autopolimerización, en resina de fotopolimerización, en compómero, liberadores de flúor y sellantes polimerizados por luz UV ${ }^{(3)}$. Si bien, aún no hay datos sobre qué material tiene un mejor efecto preventivo(1), la evidencia indica que la tasa de retención (83.9\% a los 5 años) de los sellantes basados en resina de fotopolimerización es superior al resto de los materiales mencionados ${ }^{(1)}$. Una nueva tendencia en el desarrollo de biomateriales dentales resinosos son los materiales autoadhesivos, los cuales se basan en moléculas de metacrilatos convencionales pero que además incorporan monómeros acídicos, que usualmente están presentes en los adhesivos dentinarios, que son capaces de generar una adhesión micromecánica, y posiblemente química, al interactuar con los tejidos dentarios ${ }^{(4)}$. Las resinas autoadhesivas han sido desarrolladas para utilizarse como agentes cementantes ${ }^{(5)}$, sellantes ${ }^{(6)}$ $y$, más recientemente, como resinas compuestas fluidas ${ }^{(4)}$. De estas últimas, Fusio Liquid Dentin $®$ (Pentron Clinical) es una de las pocas resinas fluidas autoadhesivas disponibles en el mercado, compuesta de

1. Cirujano Dentista. Docente del Departamento de Odontología Restauradora, Universidad Andrés Bello. Viña del Mar, Chile.

2. Magíster en Odontología Restauradora. Docente del Departamento de Odontología Restauradora, Universidad Andrés Bello. Viña del Mar, Chile.

3. Cirujano Dentista. Chile.

4. Magíster en Odontología Restauradora. Jefe del Departamento de Odontología Restauradora, Universidad Andrés Bello. Viña del Mar, Chile.

Correspondencia autor: Diego De Nordenflycht. diego.den@gmail.com. Departamento de Odontología Restauradora, Universidad Andrés Bello. Viña del Mar, Chile. Los resultados preliminares de esta investigación fueron presentados en la Sesión General de la IADR en Foz de Iguazú, Brasil, 20-23 de Junio de 2012. Trabajo recibido el 03/07/2012. Aprobado para su publicación el 05/11/2012. 
una mezcla de resinas (UDMA, TEGDMA, HEMA y 4-META), partículas de vidrio de $1.2 \mu \mathrm{m}$ (65\% en peso, $52 \%$ en volumen), estabilizadores, pigmentos orgánicos e inorgánicos y opacificadores, y que dentro de sus indicaciones clínicas está la utilización como sellante preventivo de fosas y fisuras. Sin embargo, a la fecha no existen publicaciones independientes sobre su capacidad de sellado en fosas y fisuras de esmalte humano. Por lo tanto, el objetivo de la presente investigación es evaluar la capacidad de sellado de la resina fluida autoadhesiva Fusio Liquid Dentin, mediante un test de microinfiltración in vitro, y compararla con la de un sellante de resina convencional.

\section{MATERIALES Y MÉTODO}

Se utilizaron 140 terceros molares definitivos, humanos, incluidos, sanos, recientemente extraídos, los cuales fueron almacenados en suero fisiológico $0.9 \%$, renovado cada semana desde la exodoncia hasta la etapa experimental. A todos los dientes se les removió el material orgánico mediante instrumentación periodontal manual (J30-33 JK5, American Eagle, Missoula, EE.UU.) y fueron limpiados con escobilla de profilaxis a baja velocidad con agua potable del grifo. Posteriormente, las raíces de los dientes fueron sellados con cemento de Cianoacrilato (La Gotita ${ }^{\circledR}$, Akapol, Buenos Aires, Argentina) y 3 capas de esmalte para uñas (Maybelline $®$, L'Oreal, Nueva York, EE.UU.), con el objetivo de evitar la microinfiltración no deseada. Para facilitar la manipulación y posterior montaje de las muestras, las raíces fueron incluidas en cubos de acrílico de autocurado (Acrílico Rosado, Marche, Santiago, Chile). Luego, las superficies oclusales de los dientes fueron sometidas a profilaxis con escobilla de copa blanda a baja velocidad durante 20 segundos. La escobilla fue renovada cada 15 dientes. Posteriormente, los dientes fueron distribuidos aleatoriamente en cuatro grupos $(n=35)$ y cada grupo recibió un acondicionamiento previo de la superficie y un procedimiento de sellado de las fosas y fisuras con un biomaterial sellante. Se establecieron los siguientes grupos:

- Grupo 1 (CP): Acondicionamiento con ácido fosfórico 35\% (Scotchbond

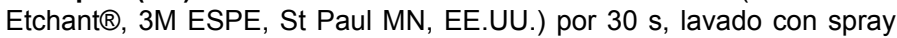
de agua por $20 \mathrm{~s}$, secado con chorro de aire por $10 \mathrm{~s}$, aplicación de sellante (Clinpro®, 3M ESPE, St. Paul MN, EE.UU.), el cual se dispensó y distribuyó con la punta aplicadora por la totalidad de las fosas y fisuras, fotopolimerización por $20 \mathrm{~s}$ con lámpara de fotocurado a $600 \mathrm{~mW} / \mathrm{cm}^{2}$ (Radii-Cal@, SDI, Victoria, Australia).

- Grupo 2 (AF+FLD): Acondicionamiento con ácido fosfórico $35 \%$ por $30 \mathrm{~s}$, lavado con spray de agua por $20 \mathrm{~s}$, secado con chorro de aire por $10 \mathrm{~s}$, aplicación de resina fluida autoadhesiva (Fusio Liquid Dentin®, Pentron Clinical, Orange CA, EE.UU.), la cual se dispensó y distribuyó con la punta aplicadora por la totalidad de las fosas y fisuras, fotopolimerización por $20 \mathrm{~s}$ con lámpara de fotocurado a $600 \mathrm{~mW} / \mathrm{cm}^{2}$ (Radii-Cal®, SDI, Victoria, Australia).

- Grupo 3 (FLD): Aplicación de resina fluida autoadhesiva sin acondicionamiento previo, la cual se dispensó y distribuyó con la punta aplicadora por la totalidad de las fosas y fisuras, fotopolimerización por $20 \mathrm{~s}$ con lámpara de fotocurado a $600 \mathrm{~mW} / \mathrm{cm}^{2}$ (Radii-Cal@, SDI, Victoria, Australia).

- Grupo 4 (MA+FLD): Acondicionamiento con microarenado (UltraBlaster $囚$, Ultradent, South Jordan UT, EE.UU.) con partículas de óxido de aluminio de $50 \mu \mathrm{m}$ a $10 \mathrm{~mm}$ de distancia por $10 \mathrm{~s}$ a 80 psi de presión, lavado con spray de agua por $20 \mathrm{~s}$ y secado con chorro de aire por $10 \mathrm{~s}$, aplicación de resina fluida autoadhesiva, la cual se dispensó y distribuyó con la punta aplicadora por la totalidad de las fosas y fisuras, fotopolimerización por $20 \mathrm{~s}$ con lámpara de fotocurado a $600 \mathrm{~mW} / \mathrm{cm}^{2}$.

Los sellantes fueron evaluados en cuanto a integridad superficial y ajuste marginal por método visual y táctil, controlando que se encontrara cubriendo todas las fosas y fisuras, y la sonda no presentara retención en los bordes. Los dientes sellados fueron termociclados por 500 ciclos con esquema de $30 \mathrm{~s}$ a $5^{\circ} \mathrm{C}$ y $30 \mathrm{~s}$ a $55^{\circ} \mathrm{C}$, con intervalos de 5 s para las transiciones (Heating Baht B-490, Büchi, Flawil, Suiza). Se preparó una tinción de nitrato de plata amoniacal con $13 \mathrm{~g}$ de nitrato de plata, $13 \mathrm{ml}$ de agua destilada y amoniaco al $25 \%$, la que luego, se disolvió en agua destilada hasta alcanzar una concentración del $50 \%$ $(\mathrm{pH}=14)$. Los dientes fueron inmersos en esta solución por 24 horas en completa oscuridad. Luego fueron lavados con agua destilada por 1 minuto y sumergidas en solución reveladora de radiografías durante 8 horas (Kodak GBX®, Eastman Kodak Company, Nueva York, EE.UU.). Posteriormente, los dientes fueron cortados (Isomet 1000, Buehler, Lake Bluff IL, EE.UU.) en sentido axial en dirección vestíbulo-lingual, perpendicular a la superficie oclusal, y se obtuvieron 2 láminas de 1.5 $\mathrm{mm}$ de grosor de la porción central del surco principal. Todas las láminas fueron pulidas manualmente, con lija al agua grano 500 y 1200 , en movimientos circulares y presión constante. Se observaron 4 superficies por cada diente en un microscopio estereoscópico a un aumento de 4x (SZ51, Olympus, Hamburgo, Alemania). Se obtuvieron imágenes digitales, que fueron evaluadas por un sólo observador, entrenado en estudio piloto de microinfiltración con 20 imágenes (kappa=0.95). Para las mediciones digitales se utilizó el software Universal Desktop Ruler (Avpsof, USA). Se analizaron dos variables para cada imagen: porcentaje de microinfiltración (definido como $A+C / B+D * 100$; ver Figura 1) y porcentaje de penetración en la fisura (definido como $A / B^{*} 100$; ver Figura 2). Los resultados fueron analizados con test ANOVA para evaluar la influencia del acondicionamiento y biomaterial sellante en el porcentaje microinfiltración y en el porcentaje de penetración en la fisura, y se realizaron comparaciones múltiples mediante el test post hoc de Dunnett con el software SPSS 20 (IBM Corporation, Chicago IL, EE.UU.) para cada variable. El nivel de significancia fue establecido como $p=0.05$.

\section{RESULTADOS}

\section{Porcentaje de Microinfiltración}

La Figura 3 muestra la distribución de valores de porcentaje de microinfiltración por cada grupo. La Tabla 1 muestra los estadísticos descriptivos por cada grupo, observándose los valores más bajos de

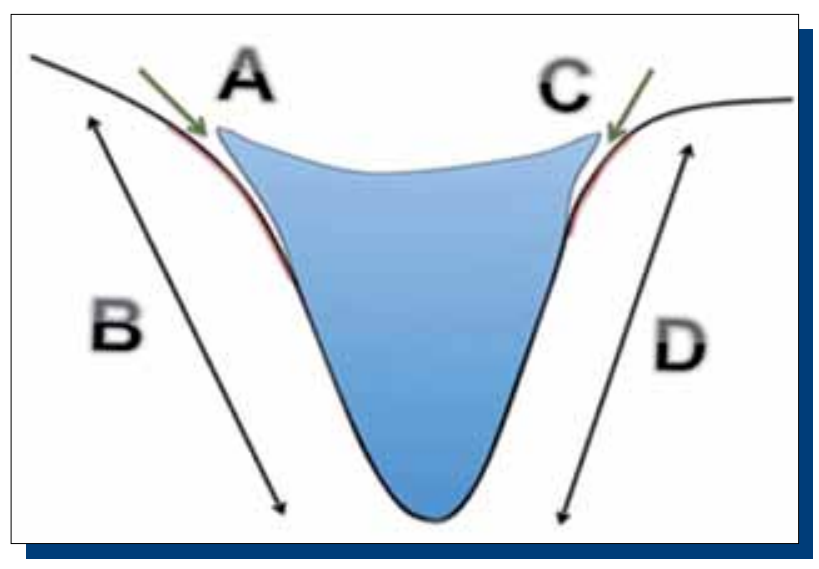

Figura 1. Esquema de mediciones para cálculo de la variable porcentaje de microinfiltración. A y C corresponden a la longitud de penetración de la tinción desde la superficie del sellante desde los extremos laterales; B y D corresponden a la longitud del sellante desde la superficie hasta el punto más profundo en ambos lados de la imagen.

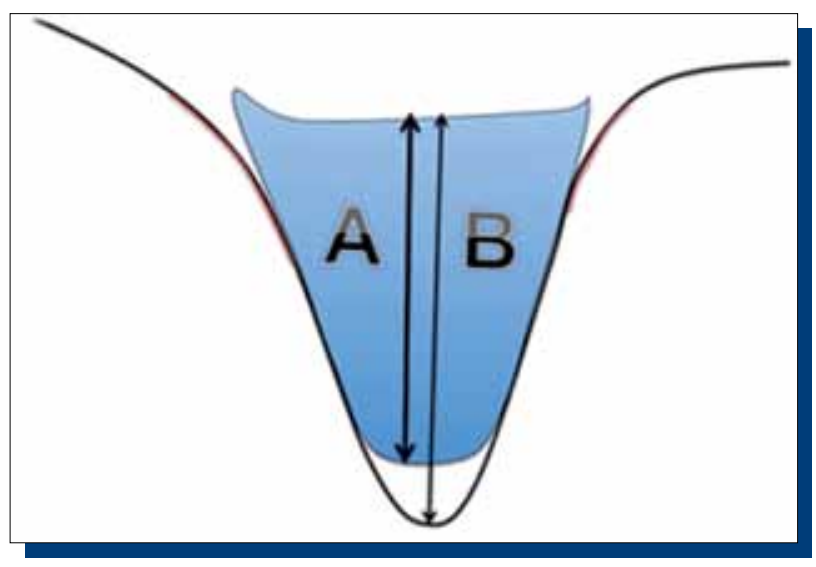

Figura 2. Esquema de mediciones para cálculo de la variable porcentaje de penetración en la fisura. A corresponde a la longitud del punto medio del sellante hasta el punto más profundo y $\mathrm{B}$ a la longitud desde el punto medio del sellante hasta el fondo de la fisura. 
microinfiltración en el Grupo 1 con una media de 13.18\%. Se observaron diferencias estadísticamente significativas entre los porcentajes de microinfiltración $[F(3,136)=963.207, p=0.000]$. Las comparaciones múltiples con test de Dunnett muestran que el Grupo 1 obtuvo una microinfiltración significativamente menor que la del Grupo $2(p=0.000)$, Grupo $3(p=0.000)$ y Grupo $4(p=0.000)$.

\section{Porcentaje de Penetración en la Fisura}

La Figura 4 muestra la distribución de valores de penetración del sellante en las fisuras por cada grupo. La mayor penetración se observó en el Grupo 1, donde 30 dientes fluctuaron entre un $98 \%$ a $100 \%$ de penetración en la fisura. La menor penetración fue para el grupo 4 , donde 20 dientes fluctuaban entre un $90 \%$ a $100 \%$. La Tabla 2 muestra los estadísticos descriptivos por cada grupo. La media de penetración más alta se observó en el Grupo $1(98.92 \%)$ y la más baja en el Grupo 4 (89.32\%). Se observaron diferencias estadísticamente significativas entre los porcentajes de penetración en al fisura $[F(3,136)=3.421, p=0.019]$. Las comparaciones múltiples con test de Dunnett (Grupo 1 como control) muestran que el Grupo 1 obtuvo una penetración significativamente mayor que la del Grupo $4(p=0.005)$. Las diferencias con los Grupos 2 y 3 no fueron significativas $(p>0.05)$.

Tabla 1. Descriptivos de la variable porcentaje de microinfiltración.

\begin{tabular}{|l|c|c|c|c|c|}
\hline & Mínimo & Máximo & Media & Mediana & $\begin{array}{c}\text { Desviación } \\
\text { Estándar }\end{array}$ \\
\hline Grupo 1 (CP) & 2.74 & 40.14 & 13.18 & 9.95 & 9.25 \\
\hline Grupo 2 (AF+FLD) & 60.33 & 100 & 87.87 & 92.78 & 11.76 \\
\hline Grupo 3 (FLD) & 86.66 & 100 & 99.08 & 100 & 2.80 \\
\hline Grupo 4 (MA+FLD) & 78.09 & 100 & 98.27 & 100 & 4.69 \\
\hline
\end{tabular}

Valores corresponden a porcentajes (\%).

Tabla 2. Descriptivos de la variable porcentaje de penetración en la fisura.

\begin{tabular}{|l|c|c|c|c|c|}
\hline & Mínimo & Máximo & Media & Mediana & $\begin{array}{c}\text { Desviación } \\
\text { Estándar }\end{array}$ \\
\hline Grupo 1 (CP) & 87.16 & 100 & 98.92 & 100 & 2.77 \\
\hline Grupo 2 (AF+FLD) & 36.45 & 100 & 93.38 & 100 & 14.26 \\
\hline Grupo 3 (FLD) & 63.72 & 100 & 94.12 & 100 & 10.46 \\
\hline Grupo 4 (MA+FLD) & 39.47 & 100 & 89.32 & 100 & 17.70 \\
\hline
\end{tabular}

Valores corresponden a porcentajes (\%).

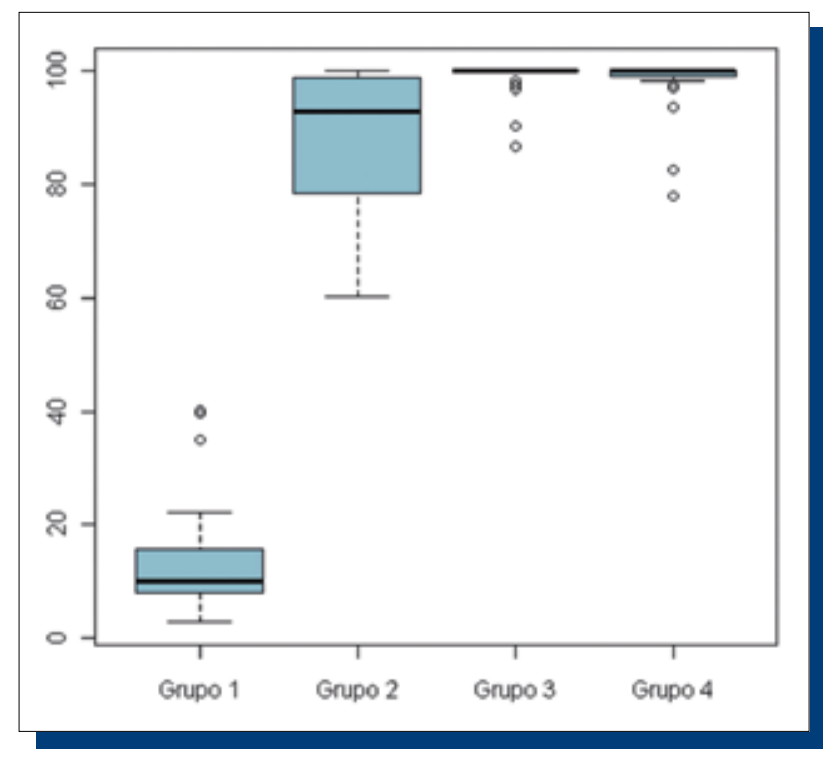

Figura 3. Distribución de valores para la variable porcentaje de microinfiltración.

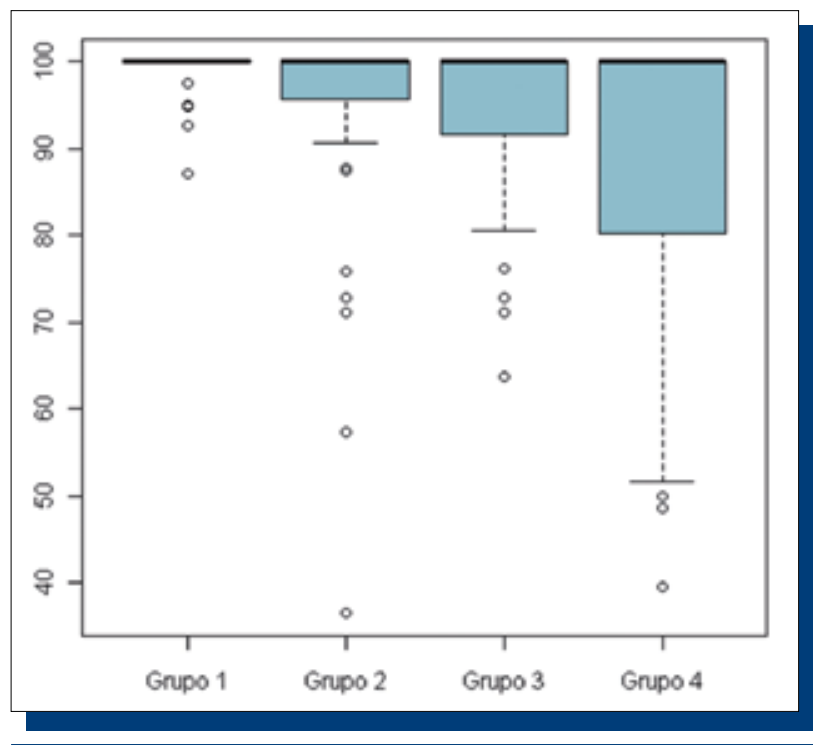

Figura 4. Distribución de valores para la variable porcentaje de penetración en la fisura.

\section{DISCUSIÓN}

La eficacia de los sellantes en la prevención de caries depende de varios factores, entre los que se encuentran la retención a largo plazo, la integridad marginal y la técnica de aplicación ${ }^{(6)}$. Una buena capacidad de sellado y la retención en el esmalte son vitales para el éxito de los sellantes de fosas y fisuras. La microinfiltración en los márgenes del sellante puede llevar a la acumulación de bacterias y a un aumento en la probabilidad de desarrollar una lesión de caries $^{(7)}$. Dado que los sellantes son frecuentemente aplicados en dientes recientemente erupcionados en niños que no siempre tienen un comportamiento adecuado al momento de la atención clínica, surge la necesidad de desarrollar nuevas técnicas y materiales que minimicen el tiempo clínico y los errores en la aplicación del sellante, como el grabado excesivo del esmalte y la contaminación con saliva ${ }^{(6)}$. Debido a esto, las resinas autoadhesivas aparecen como una alternativa atractiva para sobrellevar la sensibilidad de la técnica clínica del sellante de resina convencional. Actualmente, existen pocos materiales autoadhesivos diseñados con el propósito de actuar como sellante de fosas y fisuras. El sellante autoadhesivo con mayor cantidad de publicaciones reportadas es Enamel Loc (Premier Dental), diseñado con el objetivo de eliminar el grabado ácido, lavado y secado, sin embargo, estudios in vitro han demostrado que este material tiene pobre resistencia adhesiva ${ }^{(6,8)}$ y logra peor sellado comparado con sellantes convencionales ${ }^{(7,9,10,11)}$.

En el presente estudio se evaluó la capacidad de sellado de Fusio Liquid Dentin, una resina fluida autoadhesiva indicada para utilización como sellante de fosas y fisuras, liner bajo restauraciones adhesivas y como material obturador en cavidades clase I, III y V. La propiedad de "autoadhesión" de esta resina la otorgan los monómeros acídicos incorporados que son capaces de acondicionar el tejido dentario sin la utilización previa de un ácido fuerte inorgánico (de la misma forma que los adhesivos autograbantes). En nuestra revisión de la literatura no se encontraron publicaciones independientes acerca del comportamiento clínico de este biomaterial. Sólo se encontraron investigaciones publicadas por el fabricante (Pentron Clinical), lo que hace que su validez externa sea a lo menos cuestionable. La única publicación independiente que reportaba la utilización de Fusio Liquid Dentin es un estudio in vitro que evaluó la infiltración del material utilizado como sellado coronario post-endodoncia, mostrando que su capacidad de sellado se reduce con la profundidad de la obturación y genera gran infiltración versus MTA ${ }^{(12)}$. Respecto a otra resina fluida autoadhesiva disponible en el mercado, Vertise Flow, escasos estudios han reportado su comportamiento in vitro en esmalte ${ }^{(13,14)}$. Los resultados de estos estudios indican que esta resina autoadhesiva logra pobre resistencia adhesiva posterior al termociclado ${ }^{(14)}$ e infiltración similar a una resina fluida convencional ${ }^{(13)}$.

Nuestros resultados indican que Fusio Liquid Dentin tiene pobre capacidad de sellado comparado con un sellante convencional 
de resina de fotopolimerización, independiente del acondicionamiento previo de la superficie del esmalte. El sellante convencional logró significativamente menor microinfiltración marginal (ver Figura 3) y mayor penetración en la fisura (ver Figura 4), comparado con los grupos donde se utilizó Fusio Liquid Dentin. En los Grupos del 2 al 4 la superficie del esmalte fue tratada, previo a la aplicación de Fusio Liquid Dentin, siguiendo las sugerencias del mismo fabricante, es decir, sin acondicionamiento previo, con grabado ácido o con microarenado de la superficie. Las diferencias observadas entre los biomateriales evaluados podrían deberse a dos factores: La pobre capacidad de grabado del esmalte y/o la mayor viscosidad de la resina autoadhesiva. Se ha demostrado que los materiales autograbantes poseen una acidez que es insuficiente para acondicionar de manera adecuada el esmalte dental ${ }^{(15)}$ y además, como resultado de las interacciones químicas entre el esmalte y la resina, generan importantes cantidades de agua en la interfaz dienteresina. Estas moléculas de agua forman alteraciones en la capa de resina polimerizada conocidas como "árboles de agua" por su morfología distinguible con microscopía electrónica, fenómeno que provoca menor resistencia adhesiva ${ }^{(15)}$ y mayor infiltración marginal(16). Debido a esto es que algunos autores sugieren el grabado ácido de la superficie del esmalte previo a la utilización de un material autograbante ${ }^{(15)}$.

Si bien estudios clínicos han reportado que la tasa de retención de resinas fluidas utilizadas como sellante de fosas y fisuras es similar a la de un sellante convencional en dientes permanentes, estos resultados probablemente se deban a la utilización de un adhesivo de grabado y lavado previo a la aplicación de la resina fluida ${ }^{(17,18)}$. Esto sugiere que el problema de infiltración observado en nuestra investigación para Fusio Liquid Dentin, probablemente, no se deba a la indicación de utilizar una resina fluida como sellante sino al material utilizado propiamente tal. Desde un punto de vista clínico, el sellante convencional debiera ser elegido por sobre la resina fluida autoadhesiva al momento de seleccionar el material y técnica de un sellante de fosas y fisuras.

\section{CONCLUSIÓN}

Considerando las limitaciones propias de un estudio in vitro, podemos concluir que la capacidad de sellado de la resina fluida autoadhesiva Fusio Liquid Dentin es inferior a la de un sellante convencional de resina.

\section{CONFLICTO DE INTERESES}

Los autores declaran no tener ninguna vinculación comercial ni financiera con cualquiera de los productos utilizados para la realización de esta investigación. La presente investigación fue realizada mediante autofinanciamiento.

\section{REFERENCIAS BIBLIOGRÁFICAS}

1. Ahovuo-Saloranta A, Hiiri A, Nordblad A, Mäkelä M, Worthington HV. Pit and fissure sealants for preventing dental decay in the permanent teeth of children and adolescents. Cochrane Database Syst Rev, 2008 Oct 8; 4: CD001830.

2. Cueto El, Buonocore MG. Sealing of pit and fissures with an adhesive resin: Its use in caries prevention. J Am Dent Assoc, 1967; 75: 121-128.

3. Kühnisch J, Mansmann U, Heinrich-Weltzien R, Hickel R. Longevity of materials for pit and fissure sealing-results from a meta-analysis. Dent Mater, 2012; 28: 298303.

4. Ferracane JL. Resin composite-state of the art. Dent Mater, 2011; 27: 29-38.

5. Radovic I, Monticelli F, Goracci C, Vulicevic ZR, Ferrari M. Self-adhesive resin cements: A literature review. J Adhes Dent, 2008; 10: 251-258.

6. Wadenya RO, Yego C, Blatz MB, Mante F. Bond strength and microleakage of a new self-etch sealant. Quintessence Int, 2009; 40: 559-563.

7. Parco TM, Tantbirojn D, Versluis A, Beiraghi S. Microleakage of self-etching sealant on noncontaminated and saliva-contaminated enamel. Pediatr Dent, 2011; 33: $479-483$.

8. Guven $Y$, Tuna EB, Aktoren $O$. Shear bond strengths of self-etching and conventional fissure sealants. J Dent Res, 2007; 86(Spec Iss B): 226. Disponible en: "www.dentalresearch.org". Acceso 30 de Mayo de 2012.

9. Harz D, Urzúa I, Córdova C, Fresno MC. Estudio in vitro de la microfiltración de un sellante de autograbado. Rev Clin Periodoncia Implantol Rehabil Oral, 2009; 2 148-151.

10. Perdigão J, Sezinando A, Gomes G. In vitro sealing potential of a self-adhesive pit and fissure sealant. Quintessence Int, 2011; 42: e65-73.
11. Conner F, Vandewalle $\mathrm{K}$, Bartoloni J. Microleakage of a new self-etching sealant. J Dent Res, 2009; 88 (Spec Iss A): 495.

12. Ghulman MA, Gomaa M. Effect of intra-orifice depth on sealing ability of four materials in the orifices of root-filled teeth: An ex-vivo study. Int J Dent, 2012; 2012: 318108

13. Rengo C, Goracci C, Juloski J, Chieffi N, Giovannetti A, Vichi A, Ferrari M. Influence of phosphoric acid etching on microleakage of a self-etch adhesive and a self-adhering composite. Aust Dent J, 2012; 57: 220-226.

14. Goracci C, Margvelashvili M, Giovannetti A, Vichi A, Ferrari M. Shear bond strength of orthodontic brackets bonded with a new self-adhering flowable resin composite. Clin Oral Investig, 2013; 17: 609-617.

15. Van Meerbeek B, Yoshihara K, Yoshida Y, Mine A, De Munck J, Van Landuyt KL. State of the art of self-etch adhesives. Dent Mater, 2011; 27: 17-28.

16. Perdigão J, Monteiro P, Gomes G. In vitro enamel sealing ability of self-etch adhesives. Quintessence Int, 2009; 40: 225-233.

17. Corona SA, Borsatto MC, Garcia L, Ramos RP, Palma-Dibb RG. Randomized controlled trial comparing the retention of a flowable restorative system with a conventional resin sealant: One-year follow up. Int J Paediatr Dent, 2005; 15: 44-50. 18. Jafarzadeh M, Malekafzali B, Tadayon N, Fallahi S. Retention of a flowable composite resin in comparison to a conventional resin-based sealant: One-year follow-up. J Dent (Tehran), 2010; 7: 1-5. 